\title{
OPEN Production of genome-edited Daphnia for heavy metal detection by fluorescence
}

Takuto Arao ${ }^{1}$, Yasuhiko Kato ${ }^{1,2}$, Quang Dang Nong $^{1}$, Hiroshi Yamamoto ${ }^{3}$, Haruna Watanabe Tomoaki Matsuura ${ }^{1}$, Norihisa Tatarazako ${ }^{4}$, Kazune Tani ${ }^{5}$, Akira Okamoto ${ }^{5}$, Takeru Matsumoto ${ }^{5}$ \& Hajime Watanabe ${ }^{1 \bowtie}$

Aquatic heavy metal pollution is a growing concern. To facilitate heavy metal monitoring in water, we developed transgenic Daphnia that are highly sensitive to heavy metals and respond to them rapidly. Metallothionein A, which was a metal response gene, and its promoter region was obtained from Daphnia magna. A chimeric gene fusing the promoter region with a green fluorescent protein (GFP) gene was integrated into $D$. magna using the TALEN technique and transgenic Daphnia named $D$. magna MetalloG were produced. When $D$. magna MetalloG was exposed to heavy metal solutions for $1 \mathrm{~h}$, GFP expression was induced only in their midgut and hepatopancreas. The lowest concentrations of heavy metals that activated GFP expression were $1.2 \mu \mathrm{M} \mathrm{Zn}^{2+}, 130 \mathrm{nM} \mathrm{Cu}^{2+}$, and $70 \mathrm{nM} \mathrm{Cd}^{2+}$. Heavy metal exposure for $24 \mathrm{~h}$ could lower the thresholds even further. $D$. magna MetalloG facilitates aqueous heavy metal detection and might enhance water quality monitoring.

Excessive amounts of heavy metals are hazardous to human and ecological health. Heavy metals are discharged from industrial and natural sources. They are major aquatic toxicants, so the concern around heavy metal pollution in water is increasing in recent years. The maximum allowable metal concentrations in environmental and drinking water are strictly regulated in many countries. For regulation of aquatic heavy metal, many monitoring methods have been developed ${ }^{1}$, including instrumental analyses such as flame atomic absorption spectrometry, electrothermal atomic absorption spectrometry, inductively coupled plasma optical emission spectrometry, and inductively coupled plasma mass spectrometry ${ }^{2}$. Nevertheless, these techniques are expensive and time-consuming; therefore, simple, rapid, and efficient methods are desired for continuous heavy metal monitoring (REF).

Biomonitoring is one of the simplest ways to detect toxicants, i.e., evaluating toxicity based on biological responses. Many organisms such as algae, zooplankton, insects, and fish have been used as biomonitors for aquatic pollutants in the past ${ }^{3}$. In addition to these conventional species, frogs has also been studied as a target species for heavy metals ${ }^{4}$. However, advances in genetic engineering have changed the classical endpoints, because gene expression change can be used as one of the endpoints. Alterations in gene expression occur before phenotypic changes appear, with the latter utilized as classical endpoints. Recently, the use of toxicogenomics based on transcription profiles has emerged ${ }^{5}$. It detects and explains global transcription changes induced by toxicants and it is recognized as a sensitive method; however, it is time-consuming and costly. It is informative to evaluate the expression of target genes that can be activated by toxicants. Moreover, the application of green fluorescent protein (GFP) in transgenic animals facilitates detection of gene expression changes caused by environmental changes or the presence of toxicants. In these transgenic animals, GFP is designed to be induced by the promoter of an activated gene. To monitor water quality, transgenic zebrafish and medaka have been developed ${ }^{6}$ to detect specific pollutants including metals ${ }^{7}$, aromatic carbons ${ }^{8}$, and estrogens ${ }^{9}$.

Gene expression changes in response to heavy metals have been extensively investigated. Metallothionein is one of the genes activated by heavy metal exposure ${ }^{10}$. It is a small, highly conserved protein found in organisms ranging from bacteria to humans ${ }^{11}$, and is rich in cysteine residues, the thiol groups of which bind metals. Metallothionein is, therefore, be an excellent biomarker for metal exposure ${ }^{12}$. Transgenic fish carrying the red fluorescent protein gene DsRed2 under the control of the metallothionein gene have been constructed ${ }^{13}$.

\footnotetext{
${ }^{1}$ Department of Biotechnology, Graduate School of Engineering, Osaka University, Suita, Osaka, Japan. ${ }^{2}$ Frontier Research Base of Global Young Researchers, Graduate School of Engineering, Osaka University, Suita, Japan. ${ }^{3}$ Center for Health and Environmental Risk Research, National Institute for Environmental Studies, Tsukuba, Japan. ${ }^{4}$ Faculty of Agriculture, Ehime University, Matsuyama, Ehime, Japan. ${ }^{5}$ Department of Environmental Science and Toxicology, Nippon Soda Co., Ltd., Kanagawa, Japan. ${ }^{\square}$ email: watanabe@ bio.eng.osaka-u.ac.jp
} 
Metallothionein gene Ia1 obtained from green mussels was fused to DsRed2 and introduced into zebrafish. Transgenic zebrafish responded to $4.4 \mu \mathrm{M} \mathrm{Cd}, 7.9 \mu \mathrm{M} \mathrm{Cu}$, and $306 \mu \mathrm{M} \mathrm{Zn}$. The use of the metallothionein gene was also reported for Caenorhabditis elegans ${ }^{14}$, whose lowest observed effect concentrations were $5 \mu \mathrm{M}$ for Cd, $10 \mu \mathrm{M}$ for $\mathrm{Cu}$, and $250 \mu \mathrm{M}$ for $\mathrm{Zn}$. The use of transgenic animals for metal monitoring is simple; however, the detection limit is still very high.

Daphnia magna is a small freshwater crustacean that is used as an aquatic pollution bioindicator and model organism in ecotoxicology. As a rule, the adverse effects of aquatic pollutants have been evaluated using standard protocols such as OECD test guidelines 202 and 211 for acute and chronic toxicity testing, respectively. Daphnia are generally more sensitive to environmental changes than fish, and therefore it is expected that transgenic Daphnia could be used as an indicator of heavy metals. It has been reported that the metallothionein gene of D. pulex is activated by exposures to $\mathrm{Cd}^{11}$ and other heavy metals ${ }^{15}$. Therefore, the daphnid metallothionein gene might serve as a sensitive biomarker even in Daphnia. Recently, we successfully applied genome editing to Daphnia ${ }^{16-19}$ as well as conventional transgenic methods ${ }^{15}$. In the present study, we developed D. magna as a transgenic biomonitoring animal. Using the genome editing technique, we selected the metallothionein gene as a biomarker and visualized changes in its expression by linking it to GFP, thereby generating germ line transmitted transgenic Daphnia.

\section{Materials and methods}

Metal analysis. Test solution samples for chemical analysis were prepared in a plastic cup, separate from each test. The samples were collected at the start and the end of the exposure period to determine dissolved heavy metal concentrations. The collected samples were diluted using ultrapure water and nitric acid (final concentration of $0.13 \mathrm{M}$ ), spiked with an internal standard (Ga and In, the final concentration of $2 \mu \mathrm{g} / \mathrm{L}$ and $10 \mu \mathrm{g} / \mathrm{L}$ ), and then analyzed using ICP-MS (Agilent 8800, Agilent Technologies, Inc.).

Daphnia culture. D. magna (NIES clone) were obtained from the National Institute for Environmental Studies (Tsukuba, Japan). Cultures with a density of 16 individuals/L were maintained at $23 \pm 1{ }^{\circ} \mathrm{C}$ under a $14 \mathrm{~h}$ light $/ 10 \mathrm{~h}$ dark photoperiod in $5 \mathrm{~L}$ of Aachener Daphnien Medium $(\mathrm{ADaM})^{20}$. A $0.08 \mathrm{~mL}$ aliquot of a $7 \times 10^{9}$ cells/mL Chlorella suspension was added daily to each culture ${ }^{21}$.

Heavy metal exposure. For mRNA expression analysis of MT genes, neonate Daphnia less than 24 h old were used, which corresponded to $<96 \mathrm{~h}$ after oviposition because it takes $72 \mathrm{~h}$ for the neonate to swim out from the brood chamber. For GFP fluorescence analysis, juvenile D. magna MetalloG Daphnia (7 d post-oviposition) was used. Five each of neonate or juvenile daphniids were placed in $250 \mathrm{ml}$ of ADaM medium and exposed to various metal concentrations over a 24 -h or $48 \mathrm{~h}$ period. They were incubated at $23 \pm 1^{\circ} \mathrm{C}$ under a $14 \mathrm{~h} \mathrm{light} / 10 \mathrm{~h}$ dark photoperiod. Stock solutions of $\mathrm{ZnCl}_{2}, \mathrm{CuSO}_{4}$, and $\mathrm{CdCl}_{2}$ (Nacalai Tesque; Kyoto, Japan) were added to make up the final metal concentrations. Nominal concentrations were $0.59 \mu \mathrm{M}(80 \mu \mathrm{g} / \mathrm{l}), 2.9 \mu \mathrm{M}(400 \mu \mathrm{g} / \mathrm{l})$, and $14.7 \mu \mathrm{M}(2000 \mu \mathrm{g} / \mathrm{l})$ for the $\mathrm{ZnCl}_{2}$ exposure, $64 \mathrm{nM}(10.2 \mu \mathrm{g} / \mathrm{l})$ and $320 \mathrm{nM}(51 \mu \mathrm{g} / \mathrm{l})$ for $\mathrm{CuSO}_{4}$ exposure. There was no feeding during exposure to heavy metals. Exposures were performed independently in triplicate.

Quantification of mRNA. Total RNA was extracted from five daphniids using Sepasol-RNA I solution (Nacalai Tesque; Kyoto, Japan) according to the manufacturer's protocol. Around $0.5 \mu \mathrm{g}$ of total RNA was generally obtained. Concentrations of RNA were determined by A260 measured by Nanodrop. One-fifth of the total RNA was used in cDNA synthesis with $250 \mathrm{ng}$ of random primers (Invitrogen, Carlsbad, CA, USA) and 200U of SuperScript III Reverse Transcriptase (Invitrogen, Carlsbad, CA, USA). Quantitative polymerase chain reaction (qPCR) was performed with SYBR GreenER qPCR Supermix Universal (Invitrogen, Carlsbad, CA, USA) using the Mx3005P real-time (RT)-PCR system (Agilent Technologies, Santa Clara, CA, USA). PCR amplifications were performed with $200 \mathrm{nM}$ primers in triplicate using the following conditions: $2 \mathrm{~min}$ at $50{ }^{\circ} \mathrm{C}$ and $10 \mathrm{~min}$ at $95^{\circ} \mathrm{C}$, followed by a total of 40 two-temperature cycles $\left(15 \mathrm{~s}\right.$ at $95^{\circ} \mathrm{C}$ and $1 \mathrm{~min}$ at $\left.60{ }^{\circ} \mathrm{C}\right)$ in a volume of $20 \mu \mathrm{l}$. Metallothionein gene coding sequences were obtained from the GenBank database (A: KF561474.1, B: KF561476.1, and C: KF561475.1) and the primers for the qPCR were designed. The primers for the metallothionein gene quantification were Metallo-A forward: CTGTTGCCAAAACAATTGCTCA; Metallo-A reverse: CTC CAGTGGCACAAATGCAAG; Metallo-B forward: TGCATTCACCACCAGTAGCG; Metallo-B reverse: GCC AAGGTAAATGCTCTCGTGTG; Metallo-C forward: GTGCCCTCGTTGTCAAGGTG; and Metallo-C reverse: ATTGGTTCCACACGTGCAGT. The primers used to amplify L32 were the same as described previously ${ }^{22}$, whose expression was used as a control. Generally, cDNA corresponding to $30 \mathrm{ng}$ of total RNA was used and reaction was performed by using Ex-Taq (Takara Bio Inc., Siga, Japan). Metallothionein gene expressions were normalized to the ribosomal protein L32 level. For the estimation of the gene expression levels, $2^{-\Delta \Delta C \tau}$ method was used (REF:Livac). On completion of the assay, a dissociation stage was carried out for melting curve analysis.

To determine tissue-specific mRNA expression, ten individuals were used for one exposure, and they were soaked in RNA later (Thermo Fisher Scientific, Yokohama, Japan) after the exposure. They were dissected under a stereomicroscope and midgut and hepatopancreas were separated from other tissues. Total RNAs were prepared from the midgut and hepatopancreas and other tissues as described above. Both RNAs were used for qPCR using the same primers and conditions as above.

To evaluate the copy number of the mRNA, ten-fold dilution series of each plasmid DNA containing the target sequence prepared to make the standard curve.

Generation of transgenic Daphnia. To make a reporter gene that can respond to heavy metal exposure, the promoter region of MT-A was fused to the GFP gene. The MT-A gene containing the promoter region and 
coding sequence was amplified from purified genomic DNA by PCR. Daphnia genomic DNA was prepared as described preciously ${ }^{17}$. The primer sequences were TCTCAGTCCAGGTTTGGTTAGGA and TGTCTTAGT TGGGTGCCATTCTC. The amplified DNA fragment was cloned into a pCR-Blunt II-TOPO vector (Thermo Fisher Scientific, Yokohama, Japan), generating pCR-MT-A. After the confirmation of the sequence, to obtain the DNA fragment containing MT-A promoter region and its vector, MT-A open reading frame and the putative three prime untranslated regions (3'-UTR) were removed by PCR using primers GCCCTTGCTCACCAT GTTGATTGAAGATTTGGAATAGTGT and TTGTCCAAACTCATCAAGGGCGAATTCCAGCAC. The GFP coding sequence and polyA signal sequence was obtained from pAcGFP-C1 (Clontech Laboratories, Inc., CA, USA) using primers ATGGTGAGCAAGGGCGCCGA and GATGAGTTTGGACAAACCACAACTAGAATG CAGTG. By fusing these two DNA fragments using In-fusion HD Cloning Kit (Clontech Laboratories, Inc., CA, USA), GFP coding sequence and SV40 poly A signal sequence were inserted under the control of MT-A, which was designated as pCR-MTApro-GFP. As a NHEJ targeting site of TALEN, eyeless-targeting recognition sites ${ }^{17}$ was used because the site was successfully used in the previous study (REF). To fuse the eyeless site with pCRMTApro-GFP, the MT-A promoter region with GFP DNA fragment was introduced to the pCS-ey2-4XJHREH2B-GFP-ey1, which is the plasmid containing eyeless site and used in the previous study (REF: Nakanishi T., Kato Y., Matsuura T., Watanabe H., (2016) TALEN-mediated knock-in) using the In-fusion Cloning Kit using the primers AAACCTCCACTGAGACTCCGACTGGCGTAATAGCGA and GCGGCCGTTACTAGTCGG TACCCAGCTTTTGTTCC. The chimeric gene was amplified by PCR using primers TCTCAGTGGAGGTTT GGTTAGGA and ACTAGTAACGGCCGCCAGTG. TALEN target sequences, CCGGCGAGAATTCTCGGT CG and ACAACAACACCTTCGGACG were located in exon 6 of eyeless gene. The ey1 TALEN mRNAs targeting these sites were synthesized as described previously ${ }^{18}$ (Supplementary Fig. S1).

Microinjection was performed as described previously ${ }^{17,23}$. Eggs were obtained from two- to three-week-old Daphnia directly after ovulation. The eggs were placed in ice-cold M4 medium ${ }^{24}$ containing $80 \mathrm{mM}$ sucrose. The ey1 TALEN mRNA (500 ng/ $\mu \mathrm{l})$ and GFP reporter plasmids $(50 \mathrm{ng} / \mu \mathrm{l})$ were mixed with the injection marker Alexa Fluor 568 dye (Life Technologies Inc., Grand Island, NY, USA) at a final concentration of $0.01 \mu \mathrm{M}$. The microinjection was performed on ice and the injected eggs were incubated in a 96 -well chamber at $23^{\circ} \mathrm{C}$ until hatching.

The transgenic line was screened by the expression of GFP in offspring (Supplementary Fig. S1). GFP expression was observed under a Leica M165C fluorescence stereoscopic microscope (Leica Microsystems Heidelberg $\mathrm{GmbH}$, Mannheim, Germany) fitted with a 480-nm excitation filter and a 510-nm barrier filter (GFP2 filter set). To confirm the transgene, inverse PCR was performed. Approximately $2 \mu \mathrm{g}$ of genomic DNA was prepared from the transgenic animals, digested with EcoR I, and circularized by ligation using T4 DNA ligase at $4{ }^{\circ} \mathrm{C}$ for $24 \mathrm{~h}$. The PCR product using primers derived from the vector sequence (GGGAGAAAGGCGGACAGGTATC and GGTAGCTCTTGATCCGGCAAAC) was sequenced to clarify the junction sequence (Supplementary Fig. S2) after cloning into a pBlunt II-TOPO vector using an In-fusion HD cloning kit (Takara Bio Inc., Siga, Japan).

Imaging of D. magna MetalloG. GFP expression was observed under a Leica M165C fluorescence stereoscopic microscope (Leica Microsystems Heidelberg GmbH, Mannheim, Germany) fitted with a 480-nm excitation filter and a 510-nm barrier filter (GFP2 filter set). Light field and fluorescent images were recorded with a color digital camera (Leica DC500; Leica Microsystems Heidelberg GmbH, Mannheim, Germany) mounted on the microscope. GFP expression was quantified based on the protocol described previously ${ }^{25}$.

To observe endogenous GFP expression of D. magna MetalloG, eggs were isolated from brood chamber of $D$. magna MetalloG just after ovulation and placed in 96 well plate containing ADaM medium.

\section{Results and discussion}

Heavy metal analysis. In order to confirm actual concentrations of heavy metals used for the exposure, each medium was sampled and heavy metals was measured by ICP-MS. ADaM medium, which was used as a control, contained certain amount of $\mathrm{Zn}(0.1-0.15 \mu \mathrm{M})$ in addition to trace amount of $\mathrm{Mn}, \mathrm{Cu}, \mathrm{Cd}$ and $\mathrm{Pb}$ (Supplementary Table S1), which were derived from sea salt used for ADaM preparation. ADaM also contains $1.45 \mu \mathrm{M}$ Se. As mentioned above, these metals may induce GFP expression in a control medium as a background. Estimated concentrations of heavy metals by ICP-MS were generally close to nominal concentrations (Supplementary Table S2).

Metallothionein gene expression changes with heavy metal exposure. By searching the daphnia genome database (http://arthropods.eugenes.org/EvidentialGene/daphnia/), we identified three MT genes named MT-A, MT-B and MT-C. To select a metallothionein gene that could be effectively and strongly activated by heavy metals, quantitative PCR analysis was performed after exposing daphniids to $\mathrm{CuSO}_{4}$. Daphniids were subjected to $64 \mathrm{nM}$ and $320 \mathrm{nM} \mathrm{Cu}^{2+}$ for $24 \mathrm{~h}$ and the mRNA expression levels of their MT genes were evaluated by q-PCR. MT-A showed the highest response to $\mathrm{Cu}^{2+}$ whereas MT-B was only slightly but not significantly activated. MT-C did not respond to $\mathrm{Cu}^{2+}$ (Fig. 1). Daphniids were also exposed to different concentrations of $\mathrm{ZnCl}_{2}$ for $24 \mathrm{~h}$ and the expression levels of MT-A, MT-B, and MT-C were examined. Only MT-A showed $>6 \times$ activation in $3 \mu \mathrm{M}$ and $15 \mu \mathrm{M} \mathrm{Zn}^{2+}$ exposures, and the induction levels were almost the same for both concentrations. MT-B was activated only at the highest $\mathrm{Zn}^{2+}$ concentration $(15 \mu \mathrm{M})$; however, MT-C was not significantly induced even at that dose (Fig. 1).

MT-A alone showed the highest response to heavy metals. In the present study, MT-B showed weak activation and MT-C hardly respond at all to heavy metals. Our findings are comparable to those of a previous study ${ }^{12}$. The promoter regions of MT-A and MT-B have three metal response element (MRE) motifs and one MRE motif ${ }^{26,27}$, respectively. The presence of MRE in the promoter regions of Daphnia MT genes was also mentioned in another 

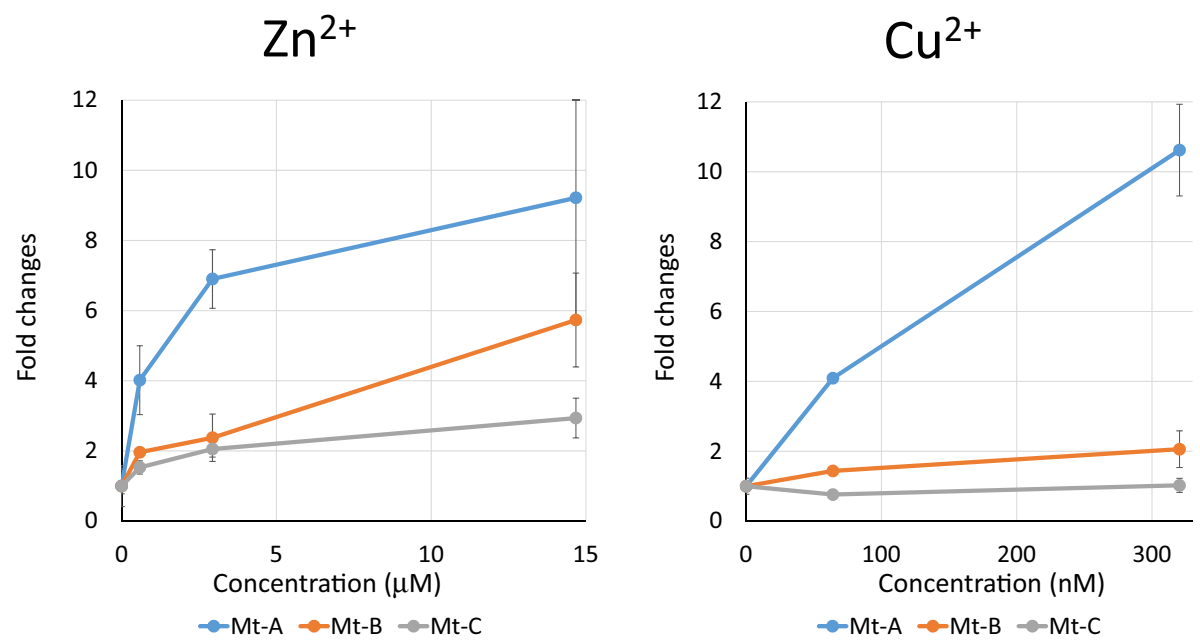

Figure 1. MT gene expression changes in response to heavy metal exposure. Indicated concentrations of $\mathrm{Zn}^{2+}$ and $\mathrm{Cu}^{2+}$ were exposed to neonate Daphnia $(<24 \mathrm{~h})$ for $24 \mathrm{~h}$. Five daphniids were used in $250 \mathrm{~mL}$ exposure and total RNA was extracted from the five daphniids. Expression levels of each MT gene were estimated by qPCR, normalized to the L32 level, and divided by the control (unexposed) expression level. The exposure was repeated three times. $\mathrm{Bar}=\mathrm{SE},{ }^{\star} p<0.05,{ }^{+} p \leq 0.1$.

study $^{15}$. Therefore, MRE might contribute to metal response and its occurrence in triplicate in MT-A might explain the fact that this metallothionein showed the strongest response to heavy metal exposures.

Transgenic Daphnia. We obtained the promoter region and 5'-UTR of MT-A to drive the GFP gene since this metallothionein displayed the strongest response to $\mathrm{ZnCl}_{2}$. We obtained $1675 \mathrm{bp}$ upstream sequence from the first ATG of MT-A and used as the MT-A promoter because coding sequence of another gene (Chitinase) was located further upstream region. We included a recognition sequence of eyeless-targeting TALEN named ey1 TALEN as previously constructed ${ }^{18}$ into the donor DNA (Supplementary Fig. S1), because we previously reported that Daphnia magna eyeless (Dma-ey, ortholog of mammalian pax6) gene can be used as a target site for recombination. This plasmid was knocked-in via TALEN-mediated non-homologous end-joining repair as described previously ${ }^{18}$. We injected 374 eggs and isolated one transgenic line designated as D. magna MetalloG (Fig. 2A, Supplementary Fig. S1). Transmission of the GFP reporter gene was confirmed by PCR using the progeny of the line and the integration site was sequenced. The transgenic Daphnia is suppsed to have $6 \mathrm{~kb}$ ( $3 \mathrm{~kb}$ of reporter gene and $3 \mathrm{~kb}$ of its vector) DNA fragment as a transgene. The junction sequence is shown in the Supporting Information (Supplementary Fig. S2).

Endogenous expression of GFP. D. magna MetalloG was cultured in ADaM and endogenous GFP expression was observed. During embryogenesis, GFP fluorescence could not be detected (data not shown). After hatching, GFP fluorescence was detected in the midgut and hepatopancreas (Fig. 2B day 1.5). The GFP fluorescence intensity in these organs was highest at day 2, then gradually decreased. At days 6 and 7, fluorescence in the midgut almost disappeared, and weak GFP expression could only be detected in the hepatopancreas (Fig. 2B). When the metal-free medium was used (M4 medium ${ }^{24}$ without any heavy metals), GFP expression could not be detected (data not shown). This result suggests that GFP expression observed at day 2 in ADaM medium was induced by the presence of metals contained in the ADaM medium itself (Supplementary Table S1). To develop a higher S/N ratio of D. magna MetalloG, we attempted to use the metal-free medium, however the D. magna MetalloG could not survive in the medium, suggesting the importance of MT-A expression or presence of heavy metals during early stages.

Tissue specific expression of MT-A and GFP. To compare the expression patterns of endogenous MT-A and GFP, D. magna MetalloG were dissected and their midgut and hepatopancreas and other tissues were separated after exposure to various concentrations of $\mathrm{Zn}^{2+}$. As shown in Fig. 3, the expression of both MT-A mRNA and GFP mRNA was exclusively observed in midgut and hepatopancreas and there was little expression in other tissues. They increased to a similar extent in a dose-dependent manner in the midgut and hepatopancreas. Therefore, the MT-A promoter used to drive GFP reflects endogenous MT-A expression well.

Although in situ hybridization is necessary to precisely compare the specific expression of endogenous MT-A and GFP transgene, our comparison of mRNA expression levels in the midgut and in other D. magna MetalloGtissues supports the fact that GFP expression mimics that of MT-A. This suggests that the promoter used in the present study contained both metal response element and DNA elements essential for tissue-specific expression in the hepatopancreas and midgut. Since these tissues are continuously exposed to the external environment, it is reasonable that the MT gene is expressed to protect cells from heavy metals existing in the external 

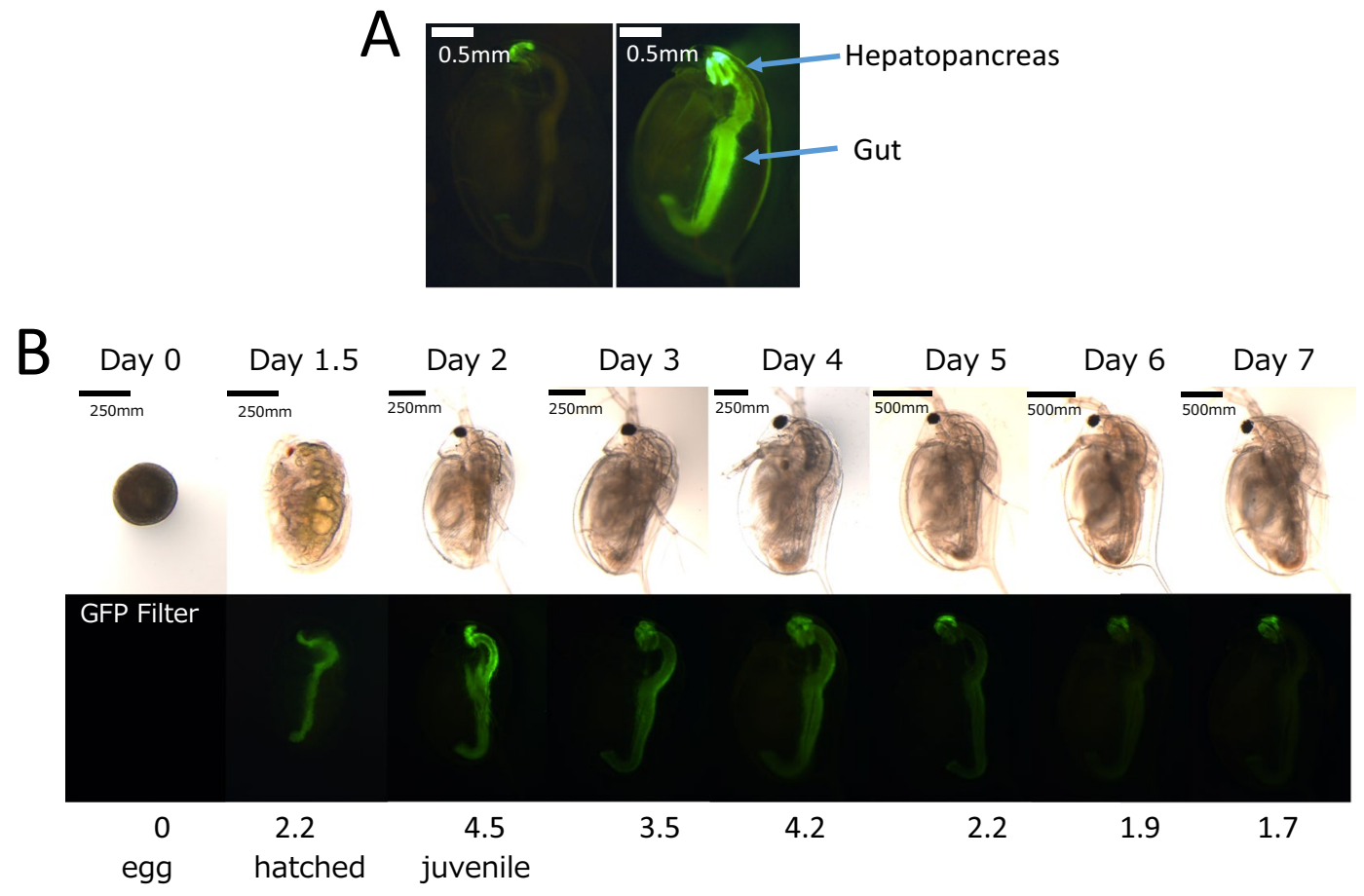

Figure 2. Heavy metal response image of D. magna MetalloG and endogenous GFP expression of D. magna MetalloG. (A) D. magna MetalloG daphnia at 7 days old exposed to $30 \mu \mathrm{M}$ of $\mathrm{ZnCl}_{2}$ for $48 \mathrm{~h}$ (right panel) and control D. magna MetalloG (unexposed) Daphnia (left panel) at the same age. (B) Eggs were obtained from D. magna MetalloG brood chamber after ovulation (assigned as Day 0) and cultured in ADaM. Pictures were taken for one week using the same parameters throughout. Bar $=250 \mu \mathrm{m}$. Relative fluorescence intensities were calculated by the method of Törner et al. (23) and indicated under the figures.

environment. It is notable that MT-A mRNA and GFP mRNA expression were comparable to the expression level of ribosomal L32 protein mRNA (Supplementary Table S3). As ribosomal proteins are ones of proteins highly expressed in cells, this result suggests the presence of abundant MT mRNA and probably MT protein in these tissues. When mRNA was prepared, a nearly tenfold induction of MT-A mRNA was observed (Fig. 1) and a greater than 20 -fold induction was observed in the isolated tissues. This higher induction of mRNA facilitates the induction and detection of GFP. Heavy metal accumulation in the hepatopancreas has been reported in crayfish $^{28}$ and heavy metal MT induction in the same organ was indicated in $\mathrm{crabs}^{29}$, which suggests that the hepatopancreas sequesters and detoxifies heavy metals in crustaceans. GFP expression of the hepatopancreas in D. magna MetalloG suggests that hepatopancreas function is widely conserved in crustacean including Daphnia and tissue functions in this organism.

GFP expression by single heavy metal exposure. Based on endogenous GFP expression, seven-dayold Daphnia was used in heavy metal exposure tests when the background GFP levels were at a minimum (Fig. 2B day 7). When D. magna MetalloG was exposed to $\mathrm{Zn}^{2+}$ for $1 \mathrm{~h}$, the GFP expression in the hepatopancreas increased. GFP expression was significantly enhanced at $\mathrm{Zn}^{2+}$ concentrations $1.2 \mu \mathrm{M}$ (Fig. 4) and GFP intensity increased with $\mathrm{Zn}^{2+}$ concentration. These results were consistent with those of the MT-A gene activation test (Fig. 1).

The responses of D. magna MetalloG to $\mathrm{Cu}^{2+}$ and $\mathrm{Cd}^{2+}$ were also examined after $1 \mathrm{~h}$ exposure. As shown in Fig. 5, GFP expression was enhanced in a dose-dependent manner in the presence of either $\mathrm{Cu}^{2+}$ or $\mathrm{Cd}^{2+}$. The minimum concentrations of $\mathrm{Cu}^{2+}$ and $\mathrm{Cd}^{2+}$ that significantly enhanced GFP expression were $130 \mathrm{nM}$ and $70 \mathrm{nM}$, respectively (Fig. 5, Supplementary Table 44 ).

To determine whether longer exposures to heavy metals reduced detection levels, we examined GFP expression changes after $24 \mathrm{~h}$. GFP expression increased with metal exposure time (Fig. 5). Significant enhancements

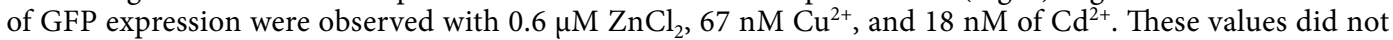
change even after $48 \mathrm{~h}$ exposure (Supplementary Table S4) and longer (Supplementary Fig. S3). These values were much lower than EC50 values of conventional chronic toxicity test for $24 \mathrm{~h}$. When EC50 values were determined using wild-type, they were as follows: $\mathrm{Zn}^{2+}: 180 \mu \mathrm{M}, \mathrm{Cu}^{2+}: 0.43 \mu \mathrm{M}$, and $\mathrm{Cd}^{2+}: 1.7 \mu \mathrm{M}$ (Supplementary Table S4). Therefore, the GFP response in transgenic Daphnia might prove useful in the detection of heavy metals.

GFP expression by multiple heavy metals exposure. To examine whether $D$. magna MetalloG responded to multiple metal exposure, $D$. magna MetalloG was exposed to $0.9 \mu \mathrm{M}$ of $\mathrm{Zn}^{2+}, 20 \mathrm{nM}$ of $\mathrm{Cu}$, or $110 \mathrm{nM}$ of $\mathrm{Cd}^{2+}$, and a combination of these metals. As shown in Fig. 6, when $\mathrm{Cd}^{2+}$ was combined with other 

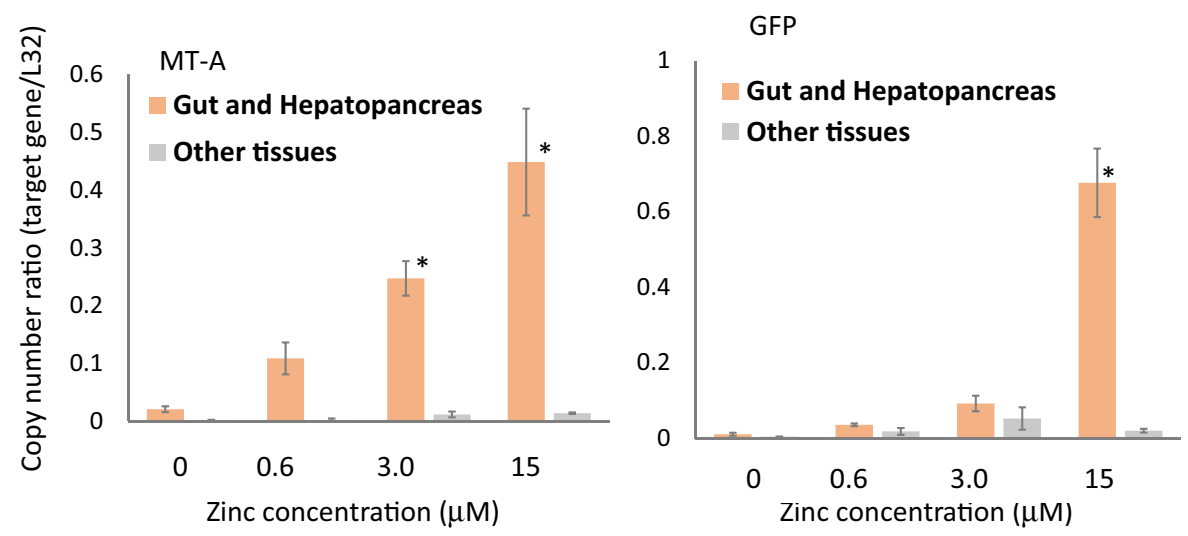

MT-A

GFP
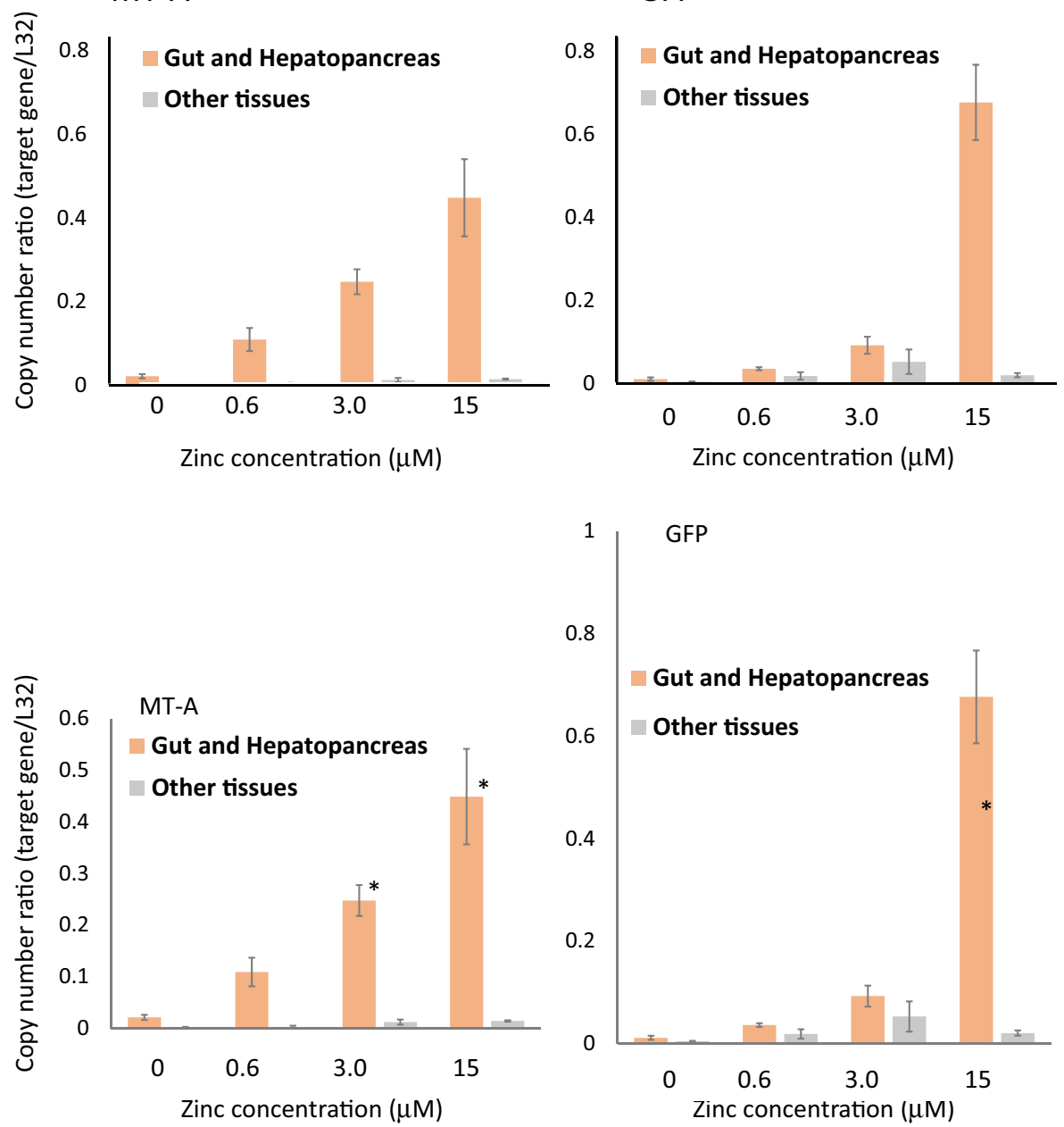

Figure 3. Tissue-specific MT-A and GFP expressions. Neonatal Daphnia $(<24 \mathrm{~h})$ were exposed to different concentrations of $\mathrm{Zn}^{2+}$ for $24 \mathrm{~h}$. Midgut and hepatopancreas of D. magna MetalloG were dissected and mRNA was purified. mRNA was also purified from the remaining tissue. The mRNA expression levels of MT-A and GFP were estimated by qPCR. Relative amounts of mRNA to that of the ribosomal L32 protein gene were estimated by $\Delta \Delta \mathrm{C} \tau$ method and indicated in $\mathrm{Y}$-axis. The quantification was performed three times. Bar $=\mathrm{SE}$.

metals, GFP fluorescence was significantly induced at $1 \mathrm{~h}$. GFP fluorescence was the highest when the three metals were mixed and exposed. Although there was no significance in the exposure of $\mathrm{Zn}^{2+}$ and $\mathrm{Cu}^{2+}$, GFP was also induced. These results indicated that GFP fluorescence could be induced additively when multiple heavy metals were exposed. After $24 \mathrm{~h}$ exposure, each metal induced GFP expression and additive effect of the mixture 

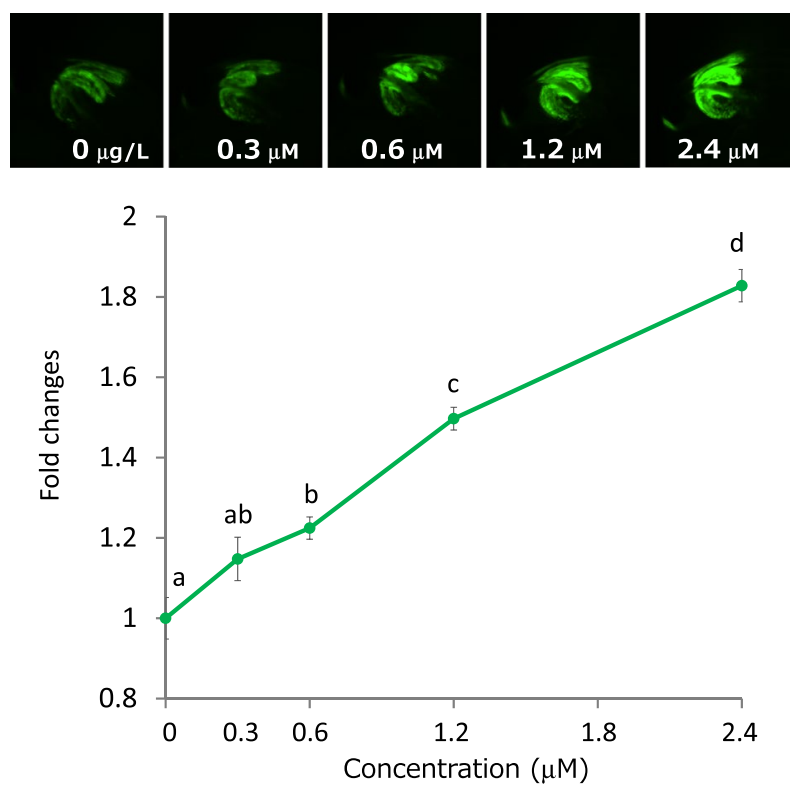

Figure 4. GFP expression changes after $\mathrm{ZnCl}_{2}$ exposure for $1 \mathrm{~h}$. Top: representative images of hepatopancreas exposed to $\mathrm{ZnCl}_{2}$ for $1 \mathrm{~h}$. Bottom: GFP expressions were quantified and fold changes vs. $0 \mathrm{mg} / \mathrm{L}$ fluorescence intensity were calculated. Five daphniids were exposed in $10 \mathrm{~mL}$ of $\mathrm{ADaM}$ for $1 \mathrm{~h}$. Fold changes vs. unexposed control were plotted. $\mathrm{N}=5 ;{ }^{* *} p<0.01$ (t-test).
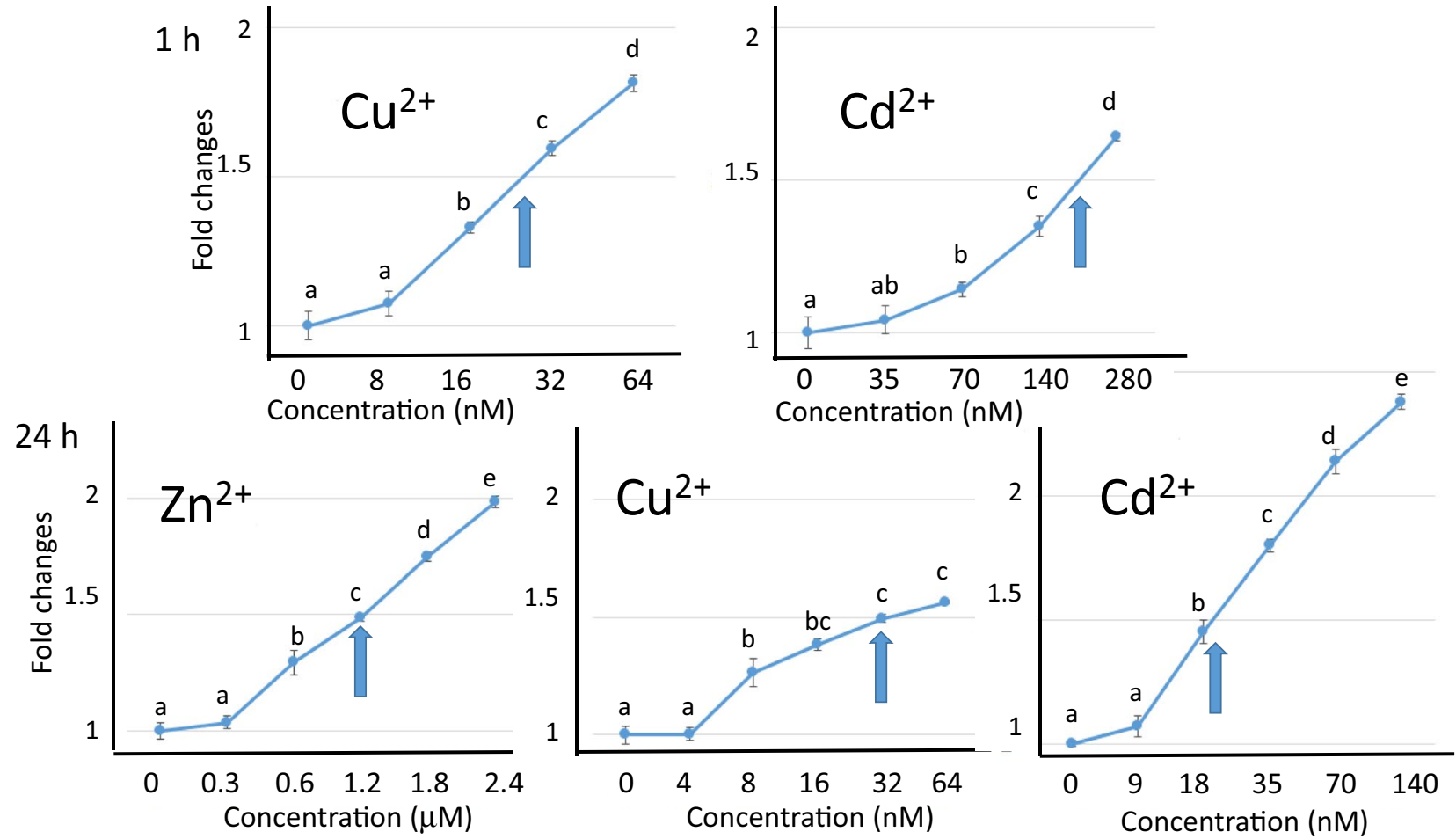

Figure 5. Dose depenent GFP expression changes after heavy metal exposure for $1 \mathrm{~h}$ or $24 \mathrm{~h}$. Five daphniids were exposed in $10 \mathrm{~mL}$ of ADaM containing indicated metal ions for $1 \mathrm{~h}$ or $24 \mathrm{~h}$. GFP expression was quantified and normalized to the control $(0 \mu \mathrm{g} / \mathrm{L})$ expression level. Numbers on $\mathrm{x}$-axis indicate concentrations of each heavy metal ( $\mu \mathrm{M}$ for $\mathrm{Zn}^{2+}$ and $\mathrm{nM}$ for $\mathrm{Cu}^{2+}$ and $\mathrm{Cd}^{2+}$ ). Y-axis indicates fold changes. Arrows indicate $\mathrm{EC}_{\mathrm{IR} 1.5}$ values. Top: $1 \mathrm{~h}$ exposure, Bottom: $24 \mathrm{~h}$ exposure. $\mathrm{N}=5 ;{ }^{* *} p<0.001 ;{ }^{* *} p<0.01 ;{ }^{*} p<0.05$ (t-test). 

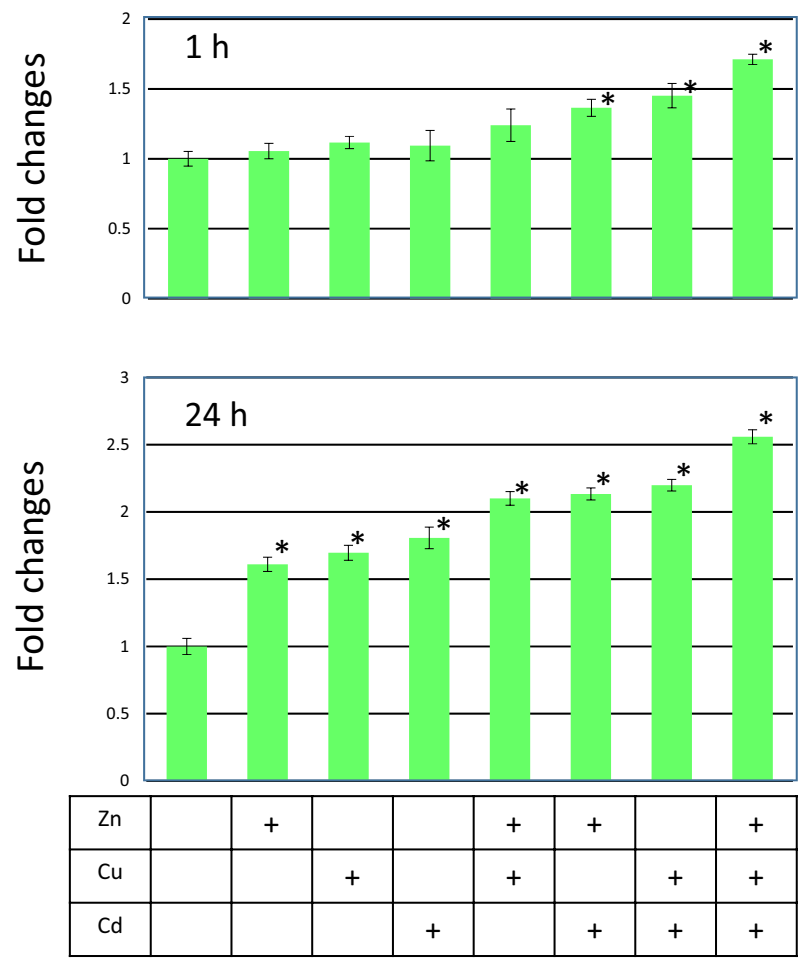

Figure 6. GFP expression by multiple heavy metals exposure. Seven day old D. magna MetalloG was exposed to $0.9 \mu \mathrm{M}$ of $\mathrm{Zn}^{2+}, 20 \mathrm{nM}$ of $\mathrm{Cu}^{2+}$, or $110 \mathrm{nM}$ of $\mathrm{Cd}^{2+}$, and a combination of these metals. After $1 \mathrm{~h}$ and $24 \mathrm{~h}$ exposure, GFP expression was quantified, These values were divided by that of control $(0 \mu \mathrm{g} / \mathrm{L})$ expression level and fold changes were calculated. Metal ions used for the exposure are indicated in the boxes (+presence of ion indicated on the left column (Y-axis indicates fold changes. ${ }^{*} p<0.05$ (t-test) Measured concentrations are indicated in Supplementary Table S5.

of metals could be prominently observed. Only one dose of the three metals were applied in the present study, additional experiments using various concentrations of metals would further clarify the GFP induction profile. Measured concentrations of the medium are indicated in Supplementary Table S5, which shows measured concentrations were close to nominal concentrations.

Advantages of $D$. magna MetalloG. The use of GFP has been proposed previously ${ }^{6}$ and applied as a reporter gene in other species. To detect heavy metals in the water, heavy metal monitoring medaka have been reported. The GFP in the transgenic medaka is driven by Hsp70, which responds to $110 \mathrm{nM} \mathrm{Cd}^{2+}$ after $24 \mathrm{~h}$ exposure $^{30}$. D. magna MetalloG can respond to $18 \mathrm{nM} \mathrm{Cd}^{2+}$ after $24 \mathrm{~h}$; therefore, the Daphnia biosensor is more sensitive than the medaka. In addition, Hsp70 can respond not only to metals but to other stimuli including heat; therefore, the expression of GFP does not necessarily indicate the presence of heavy metals. Conversely, the metallothionein gene responds to particular metals and could, therefore, be useful for the classification of toxicants. Recently, transgenic zebrafish containing the GFP gene under the metallothionein promoter have been developed; however, the transgenic zabrafish only responded to high metal concentrations $\left(\mathrm{Cd}^{2+}: 0.5 \mathrm{ppm}\right.$ $(4.5 \mu \mathrm{M}), \mathrm{Zn}^{2+}: 20 \mathrm{ppm}(306 \mu \mathrm{M})$; , and $\left.\mathrm{Cu}^{2+}: 0.3 \mathrm{ppm}(4.7 \mu \mathrm{M})\right)^{31}$. While the promoter used for the detection of heavy metal was MT, sensitivity to metals was more than hundred times higher in Daphnia. To detect heavy metals in soil, a transgenic nematode was also established ${ }^{32}$. In the transgenic nematode, the lowest observed effect concentration to $\mathrm{Cd}^{2+}$ was reported as $5 \mu \mathrm{M}(0.9 \mathrm{ppm}, 900 \mu \mathrm{g} / \mathrm{L})$. These results indicate that Daphnia is a suitable species for the detection of heavy metals in much lower concentrations, which might be reasonable because Daphnia is known to be more sensitive to heavy metals than fish. Therefore, D. magna MetalloG is the most sensitive and rapid GFP-expressing biosensor ever developed.

Compared to zebrafish and medaka, Daphnia has application advantages. Daphnia is an invertebrate; therefore, there are no legal ethical issues with this genus during experimental studies. As the regulation of animal use is becoming stricter, the application of invertebrates is preferable to vertebrates. In addition, Daphnia can mature in one week and start reproduction, which facilitates propagation and use. Based on genome editing techniques in Daphnia developed in our laboratory, this is the first reported study of a transgenic strain utilized as a biosensor strain. Our technique provides several types of biosensors using Daphnia in the near future.

Perspective of the use of D. magna MetalloG. D. magna MetalloG still has problems that need to be improved. One is endogenous expression and the other is tissue specific expression. GFP expression was detected from Daphnia's exposure to the $\mathrm{ADaM}$, which contained trace amounts of $\mathrm{Mn}, \mathrm{Rb}, \mathrm{Sr}, \mathrm{Co}$, Se, and 
other metals (Supplementary Table S1). To eliminate these heavy metal ions, we examined if metal free synthetic medium could be used for the test. When the synthetic water was used (M4 medium without any metal ions), the endogenous GFP expression was significantly reduced; however, the daphniids died. By reducing the amounts of metals in medium to minimum levels, it might be possible to decrease the lower limit of sensitivity. Exclusive GFP expression might not be desirable as a biosensor because observation of Daphnia with high magnification is required. If the Daphnia expresses GFP in their entire body, it becomes much easier to detect the expression, possibly without using a microscope. Therefore, the rational design of the promoter by eliminating the DNA element responsible for tissue-specificity and, adding multiple MER might help further development of Daphnia as a biosensor.

The detection limits to these heavy metals of D. magna MetalloGnking Water Regulations, USEPA ${ }^{33}$ Inorganic Maximum Contaminant Level Goal of Copper and Cadmium were assigned as $1.3 \mathrm{mg} / \mathrm{L}$ and $0.005 \mathrm{mg} / \mathrm{L}$, respectively. Secondary Maximal Contaminant Level of Zinc was assigned as $5 \mathrm{mg} / \mathrm{L}$ in Secondary Drinking Water Standards, USEPA ${ }^{34}$. Thus, D. magna MetalloG might be useful for detecting heavy metals near regulatory levels.

In conclusion, we developed a genome-edited Daphnia that can express GFP that responds to heavy metals. The sensitivity of GFP expression is much higher than that of conventional acute toxicity testing. In addition, genome-edited Daphnia can detect heavy metals much faster $(1 \mathrm{~h})$ than the conventional method $(24-48 \mathrm{~h})$. Daphnia is particularly sensitive to aquatic chemical stresses. By introducing a sensitive reporter gene into a susceptible organism, a highly accurate bioassay could be created. The introduction of other reporter genes responsive to other toxicants can build genome-edited Daphnia that facilitate water quality monitoring and contaminant identification.

Received: 28 July 2020; Accepted: 20 November 2020

Published online: 08 December 2020

\section{References}

1. Elzwayie, A., Afan, H. A., Allawi, M. F. \& El-Shafie, A. Heavy metal monitoring, analysis and prediction in lakes and rivers: state of the art. Environ. Sci. Pollut. Res. 24, 12104-12117 (2017).

2. Winefordner, J. D. et al. Comparing several atomic spectrometric methods to the super stars: special emphasis on laser induced breakdown spectrometry, LIBS, a future super star. J. Anal. At. Spectrom. 19, 1061 (2004).

3. Zhou, Q., Zhang, J., Fu, J., Shi, J. \& Jiang, G. Biomonitoring: An appealing tool for assessment of metal pollution in the aquatic ecosystem. Anal. Chim. Acta 606, 135-150 (2008).

4. Prokić, M. D. et al. Antioxidative responses of the tissues of two wild populations of Pelophylax kl esculentus frogs to heavy metal pollution. Ecotoxicol. Environ. Saf. 128, 21-29 (2016).

5. Iguchi, T., Watanabe, H. \& Katsu, Y. Toxicogenomics and ecotoxicogenomics for studying endocrine disruption and basic biology. Gen. Comp. Endocrinol. 153, 25-29 (2007).

6. Gong, Z., Ju, B. \& Wan, H. Green fluorescent protein (GFP) transgenic fish and their applications. Genetica 111, 213-225 (2001).

7. Blechinger, S. R., Warren, J. T., Kuwada, J. Y. \& Krone, P. H. Developmental toxicology of cadmium in living embryos of a stable transgenic zebrafish line. Environ. Health Perspect. 110, 1041-1046 (2002).

8. Ng, G. H. B. \& Gong, Z. GFP transgenic medaka (Oryzias latipes) under the Inducible cypla promoter provide a sensitive and convenient biological indicator for the presence of TCDD and other persistent organic chemicals. PLoS ONE 8, e64334 (2013).

9. Zeng, Z., Shan, T., Tong, Y., Lam, S. H. \& Gong, Z. Development of estrogen-responsive transgenic medaka for environmental monitoring of endocrine disrupters. Environ. Sci. Technol. 39, 9001-9008 (2005).

10. Klaassen, C. D., Liu, J. \& Choudhuri, S. Metallothionein: an intracellular protein to protect against cadmium toxicity. Annu. Rev. Pharmacol. Toxicol. 39, 267-294 (1999).

11. Garcia-Reyero, N. et al. Biomarker discovery and transcriptomic responses in Daphnia magna exposed to munitions constituents. Environ. Sci. Technol. 43, 4188-4193 (2009).

12. Poynton, H. C. et al. Daphnia magna ecotoxicogenomics providesmechanistic insights into metal toxicity. Environ. Sci. Technol. 80, 1044-1050 (2007).

13. Liu, L., Yan, Y., Wang, J., Wu, W. \& Xu, L. Generation of $m t$ egfp transgenic zebrafish biosensor for the detection of aquatic zinc and cadmium. Environ. Toxicol. Chem. 35, 2066-2073 (2016).

14. Ma, H., Glenn, T. C., Jagoe, C. H., Jones, K. L. \& Williams, P. L. A transgenic strain of the nematode caenorhabditis elegans as a biomonitor for heavy metal contamination. Environ. Toxicol. Chem. 28, 1311 (2009).

15. Asselman, J. et al. Functional characterization of four metallothionein genes in Daphnia pulex exposed to environmental stressors. Aquat. Toxicol. 110-111, 54-65 (2012).

16. Nakanishi, T., Kato, Y., Matsuura, T. \& Watanabe, H. CRISPR/Cas-mediated targeted mutagenesis in Daphnia magna. PLoS ONE 9, e98363 (2014).

17. Naitou, A., Kato, Y., Nakanishi, T., Matsuura, T. \& Watanabe, H. Heterodimeric TALENs induce targeted heritable mutations in the crustacean Daphnia magna. Biol. Open 4, 364-369 (2015).

18. Nakanishi, T., Kato, Y., Matsuura, T. \& Watanabe, H. TALEN-mediated homologous recombination in Daphnia magna. Sci. Rep. 5, 18312 (2015).

19. Kato, Y., Matsuura, T. \& Watanabe, H. Genomic integration and Germline transmission of plasmid injected into Crustacean Daphnia magna Eggs. PLoS ONE 7, e45318 (2012).

20. Klüttgen, B., Dülmer, U., Engels, M. \& Ratte, H. T. ADaM, an artificial freshwater for the culture of zooplankton. Water Res. 28, 743-746 (1994).

21. Kato, Y. et al. Molecular cloning and sexually dimorphic expression of DM-domain genes in Daphnia magna. Genomics 91, 94-101 (2008).

22. Kato, Y., Kobayashi, K., Watanabe, H. \& Iguchi, T. Environmental sex determination in the branchiopod crustacean Daphnia magna: deep conservation of a Doublesex gene in the sex-determining pathway. PLoS Genet. 7, e1001345 (2011).

23. Kato, Y. et al. Development of an RNA interference method in the cladoceran crustacean Daphnia magna. Dev. Genes Evol. 220, 337-345 (2011).

24. Elendt, B. P. \& Bias, W. R. Trace nutrient deficiency in Daphnia magna cultured in standard medium for toxicity testing Effects of the optimization of culture conditions on life history parameters of D. magna. Water Res. 24, 1157-1167 (1990).

25. Törner, K., Nakanishi, T., Matsuura, T., Kato, Y. \& Watanabe, H. Optimization of mRNA design for protein expression in the crustacean Daphnia magna. Mol. Genet. Genom. 289, 707-715 (2014). 
26. Stuart, G. W., Searle, P. F. \& Palmiter, R. D. Identification of multiple metal regulatory elements in mouse metallothionein-I promoter by assaying synthetic sequences. Nature 317, 828-831 (1985)

27. Westin, G. \& Schaffner, W. A zinc-responsive factor interacts with a metal-regulated enhancer element (MRE) of the mouse metallothionein-I gene. EMBO J. 7, 3763-3770 (1988).

28. Kouba, A., Buřič, M. \& Kozák, P. Bioaccumulation and effects of heavy metals in crayfish: A review. Water. Air. Soil Pollut. 211, $5-16(2010)$

29. Olafson, R. W., Kearns, A. \& Sim, R. G. Heavy metal induction of metallothionein synthesis in the hepatopancreas of the crab Scylla serrata. Comp. Biochem. Physiol. Part B Comp. Biochem. 62, 417-424 (1979).

30. Ng, G. H. B., Xu, H., Pi, N., Kelly, B. C. \& Gong, Z. Differential GFP Expression Patterns Induced by Different Heavy Metals in Tg(hsp70:gfp) Transgenic Medaka (Oryzias latipes). Mar. Biotechnol. 17, 317-327 (2015).

31. Pawar, N., Gireesh-Babu, P., Sivasubbu, S. \& Chaudhari, A. Transgenic zebrafish biosensor for the detection of cadmium and zinc toxicity. Curr. Sci. 111, 1697-1701 (2016).

32. Aschner, M. \& Martinez-Finley, E. J. Revelations from the nematode caenorhabditis elegans on the complex interplay of metal toxicological mechanisms. J. Toxicol. https://doi.org/10.1155/2011/895236 (2011).

33. National Primary Drinking Water Regulations. https://www.epa.gov/ground-water-and-drinking-water/national-primary-drink ing-water-regulations\#.

34. Secondary Drinking Water Standards: Guidance for Nuisance Chemicals. https://www.epa.gov/dwstandardsregulations/secon dary-drinking-water-standards-guidance-nuisance-chemicals.

\section{Author contributions}

T.A. made the transgenic animal and performed the main experiments. Q.D.N., K.T., A.O., T.M. took pictures and made figures. N.T. did exposure and evaluation. H.Y., H.W. measured metal concentration. T.M. and Y.K. wrote the main manuscript. H.W. supervised the experiments and finalized the manuscript. All authors reviewed the manuscript.

\section{Competing interests}

The authors declare no competing interests.

\section{Additional information}

Supplementary information is available for this paper at https://doi.org/10.1038/s41598-020-78572-z.

Correspondence and requests for materials should be addressed to H.W.

Reprints and permissions information is available at www.nature.com/reprints.

Publisher's note Springer Nature remains neutral with regard to jurisdictional claims in published maps and institutional affiliations.

(c) (i) Open Access This article is licensed under a Creative Commons Attribution 4.0 International License, which permits use, sharing, adaptation, distribution and reproduction in any medium or format, as long as you give appropriate credit to the original author(s) and the source, provide a link to the Creative Commons licence, and indicate if changes were made. The images or other third party material in this article are included in the article's Creative Commons licence, unless indicated otherwise in a credit line to the material. If material is not included in the article's Creative Commons licence and your intended use is not permitted by statutory regulation or exceeds the permitted use, you will need to obtain permission directly from the copyright holder. To view a copy of this licence, visit http://creativecommons.org/licenses/by/4.0/.

(c) The Author(s) 2020 